\title{
SCIENTIFIC REPORTS

\section{OPEN Evidence from 3-month-old infants shows that a combination of postnatal feeding and exposures in utero shape lipid metabolism}

Received: 20 June 2019

Accepted: 17 September 2019

Published online: 04 October 2019
Samuel Furse $\mathbb{C}^{1}{ }^{\text {, Stuart G. Snowden }}{ }^{1}$, Laurentya Olga $\mathbb{C}^{2}$, Philippa Prentice ${ }^{2}$, Ken K. Ong ${ }^{2,3}$, leuan A. Hughes ${ }^{2}$, Carlo L. Acerini ${ }^{2}$, David B. Dunger $\mathbb{B}^{2}$ \& Albert Koulman $\left(^{1,3}\right.$

We tested the hypothesis that both postnatal feeding and conditions in utero affect lipid metabolism in infants. Infants who experienced restrictive growth conditions in utero and others exposed to maternal hyperglycaemia were compared to a control group with respect to feeding mode. Dried blood spots were collected from a pilot subset of infant participants of the Cambridge Baby Growth Study at $3 \mathrm{mo}$. Groups: (a) a normal gestation (control, $n=40$ ), (b) small for gestational age (SGA, $n=34$ ) and (c) whose mothers developed hyperglycaemia $(n=59)$. These groups were further stratified by feeding mode; breastfed, formula-fed or received a mixed intake. Their phospholipid, glyceride and sterol fractions were profiled using direct infusion mass spectrometry. Statistical tests were used to identify molecular species that indicated differences in lipid metabolism. The abundance of several phospholipids identified by multivariate analysis, PC(34:1), PC(34:2) and PC-O(34:1), was 30-100\% higher across all experimental groups. $\mathrm{SM}(39: 1)$ was around half as abundant in in utero groups among breastfed infants only. The evidence from this pilot study shows that phospholipid metabolism is modulated by both conditions in utero and postnatal feeding in a cohort of 133 Caucasian infants, three months post partum.

There is mounting evidence that conditions in utero can have profound and long-lasting effects on infant growth and metabolism. Recent studies in animal models have indicated that maternal diet and obesity are associated with cardio-vascular dysfunction ${ }^{1,2}$, inflammation of adipose tissue ${ }^{3}$, insulin resistance ${ }^{4-6}$, adiposity ${ }^{5}$, non-alcoholic fatty liver disease ${ }^{7}$ and even modulated nutrient acquisition ${ }^{8}$ in their offspring. Exercise has also been shown to rescue obese mothers' insulin sensitivity and that of her male offspring 9 .

Similarly, a variety of exposures during pregnancy have been associated with birth outcomes in humans. It has been known for some time that factors such as smoking and a high caffeine intake during gestation lead to a lower birth weight in humans ${ }^{10-12}$. Both maternal obesity ${ }^{13-15}$ and gestational diabetes ${ }^{16-19}$ in humans typically lead to a higher birth weight, and higher rate of stillbirth ${ }^{20,21}$. Furthermore, babies small and large for gestational age are at increased risk of metabolic complications in later life ${ }^{22,23}$, which is consistent with the evidence from animal models of diet-induced obesity and gestational diabetes mellitus (GDM) $)^{1-3,5}$.

However, it is also becoming evident that dietary exposure in early life can have a profound effect on infant development in humans. Studies of the lipid profile in infant circulation show that this reflects feeding and correlates with development in the first years ${ }^{2-26}$, and hints that lipid metabolism is linked to infant development. This raises questions about how dysregulation of metabolism later in life is associated with conditions in utero, and how it might be detected early in life in order to facilitate the timing of appropriate intervention.

The importance of these questions is underscored by epidemiological evidence that the maternal hyperglycaemia (MHG) associated with GDM affects $8-24 \%$ of pregnancies in the UK, and is rising ${ }^{27}$. The concentration of

${ }^{1}$ Core Metabolomics and Lipidomics Laboratory, Metabolic Research Laboratories, Institute of Metabolic Science, University of Cambridge, Level 4 Pathology, Cambridge Biomedical Campus, Cambridge, CB2 00Q, UK. ${ }^{2}$ Department of Paediatrics, University of Cambridge, Box 116, Cambridge Biomedical Campus, Cambridge, CB2 0QQ, UK. ${ }^{3}$ MRC Epidemiology Unit, Wellcome Trust-MRC Institute of Metabolic Science, University of Cambridge, Cambridge Biomedical Campus, Cambridge, CB2 0QQ, UK. Correspondence and requests for materials should be addressed to A.K. (email: ak675@medschl.cam.ac.uk) 
glucose in the circulation of women who develop GDM is typically abnormally high due to decreased sensitivity to insulin and impaired insulin secretion. This results in higher placental transfer of glucose can contribute to more rapid growth of the foetus in utero. By contrast, there continue to be large numbers of babies who are born at a low birth weight. 'Small for gestational age' (SGA), based on sex and gestation-corrected size at birth, is often used to identify babies who had poor growth in utero. Several pregnancy-related maternal and placental factors may be involved in an infant falling into this group ${ }^{28,29}$, and such infants usually show rapid growth after birth ${ }^{30}$. Although much phenotypical data about both SGA and MHG infants has been collected, the metabolism driving these phenotypes is not clear.

This is particularly striking as several phospholipids in infant circulation correlate with their growth ${ }^{24,25}$. This raises questions about how infants of normal and high birth weight from GDM pregnancies differ, and whether the metabolism that arises from all normal-weight births are similar. The combination of the risks to health of both low- and high-weight births, the evidence that the phospholipid and triglyceride profiles in the circulation of infants is heavily dependent upon how they are fed $^{24,25}$ and correlate with their growth, and evidence that conditions in utero shape infant growth, led us to the hypothesis that the metabolic response to diet in early life is modified by exposures in utero.

To address this question, we assessed the circulating phospholipid and triglyceride profile of infants from three groups stratified by conditions in utero, and within each group we compared the mode of feeding (breastfed, formula-fed, or mixed-fed). The three in utero groups of infants included were (a) born at low birth weight for gestational age (SGA), (b) exposed to maternal hyperglycaemia (MHG) but with a normal birth weight, or (c) Controls (normal birth weight and no MHG). The groups were otherwise homogeneous, none of the mothers of SGA babies had hyperglycaemia. Each group comprised individuals who were breastfed, formula-fed or received a mixed intake. This allowed us to compare the in utero exposure across different post natal (first 3mo of life) feeding types, and also feeding types across infants exposed to different conditions in utero. This was designed to give an insight into the way both post natal (first 3 mo of life) feeding and conditions in utero affected the observed lipid metabolism at three months of age. The method for profiling the phospholipids and triglycerides present (mass spectrometry) was chosen to identify individual metabolites in order to characterise differences in lipid metabolism.

\section{Results}

Augmentation of reported data. In order to identify all possible lipid/triglyceride variables that may be associated with the phenotypical or diagnostic difference between groups, we judged that molecular species identified in both positive and negative mode should be used. We therefore augmented data from a previous study, that was based only on positive ionisation mode $\mathrm{e}^{24,25}$, with the appropriate lipid profiles from signals collected in negative ionisation mode. Statistical tests (Principal component analysis followed by T-tests) performed on this augmented dataset showed that it exhibits the same trends as the positive mode data alone; there is a clear separation between the phospholipid and glyceride profiles of the three feeding groups tested (breastfed infants, formula-fed infants and infants receiving a mixed intake), with the mixed feeding group lying between the other two (Supplementary Fig. 1A). We then examined the abundance of biomarkers of feeding identified previously in additional infants, who form the normal birth weight Control group of the current study, Supplementary Fig. 1B. This approach is used to validate the control group of this study with that used in previous work. Both the magnitude and pattern of abundance of these biomarkers of feeding type appears to be similar in this independent Control group (Supplementary Fig. 2).

Influence of feeding mode across in utero groups. The hypothesis of this study is that both postnatal feeding and conditions in utero influence the lipid metabolism observed in infants at $3 \mathrm{mo}$. In order to test this, we first stratified the participants only by the in utero groups, i.e. those who were small for gestational age (SGA), those who were exposed to maternal hyperglycaemia in utero and those of healthy pregnancies, and sought to identify the variables that distinguish the groups without reference to how the participants were fed. A Random Forest classification found no molecular species that distinguished the utero groups (SGA, MHG, Control). This suggests that feeding mode has a more pronounced influence on the lipids in circulo and thus we stratified the participants by the feeding mode as well as by conditions in utero. This approach allows us to distinguish any effects of conditions in utero from those of feeding mode.

Stratification of the participants by both feeding type and conditions in utero. The evidence that the in utero groups alone did not dominate the lipid metabolism at 3 mo led us to stratify the infants according to feeding type (breastfed, mixed, formula-fed) as well as the conditions they were experienced to in utero (giving rise to the SGA, MHG and control groups). This gave nine groups. In order to test whether lipid metabolism was different in the exposed groups (SGA, MHG), we compared exposed and control groups of the same feeding type. A supervised statistical test (sPLS-DA) was used to identify (a) the individual variables (molecular species) that distinguished, for example, the breastfed MHG group from the breastfed Control group and (b) the feeding groups within the exposed groups (e.g. breast and formula fed individuals from the MHG group). This structure was adopted in order to compare individuals who differed in either conditions in utero or by feeding mode.

Variables indicating shifts in lipid metabolism in SGA and MHG infants. None of the species identified in previous work ${ }^{25}$ was identified as a variable that drove the distinctions between different feeding types in SGA infants (Supplementary Fig. 3A, loading values in table). The lipid species that were identified as distinguishing the breastfed from formula fed SGA infants $\operatorname{SM}(36: 2)$ and $\operatorname{SM}(34: 2))$ were structurally similar to those found previously. Several lipid species distinguish MHG infants from controls (Table 1). However, three of these variables are also identified as the three most important variables for distinguishing the three feeding groups of MHG infants from one another: PC(34:2), SM(32:1) and SM(39:1) (Supplementary Fig. 3B). 


\begin{tabular}{|c|c|c|c|c|c|c|c|c|c|c|c|c|}
\hline \multirow[b]{3}{*}{ Lipid } & \multicolumn{6}{|l|}{ SGA } & \multicolumn{6}{|l|}{ MHG } \\
\hline & \multicolumn{2}{|c|}{ Breast-fed } & \multicolumn{2}{|c|}{ Mixed-fed } & \multicolumn{2}{|c|}{ Formula-fed } & \multicolumn{2}{|c|}{ Breast-fed } & \multicolumn{2}{|c|}{ Mixed-fed } & \multicolumn{2}{|c|}{ Formula-fed } \\
\hline & PC1 & PC2 & PC1 & PC2 & PC1 & PC2 & PC1 & PC2 & PC1 & PC2 & PC1 & PC2 \\
\hline $\begin{array}{l}\mathrm{PC}(34: 1) \\
\& \\
\mathrm{PE}(37: 1)\end{array}$ & 0.532 & -0.176 & 0.599 & -0.254 & 0.549 & -0.133 & 0.566 & -0.254 & 0.597 & -0.269 & 0.573 & -0.268 \\
\hline $\mathrm{PC}(34: 2)$ & 0.489 & 0.230 & 0.356 & -0.095 & 0.403 & -0.312 & 0.389 & -0.028 & 0.450 & -0.094 & 0.385 & -0.290 \\
\hline $\begin{array}{l}\mathrm{PC}(38: 4) \\
\& \\
\mathrm{PE}(41: 4)\end{array}$ & 0.041 & -0.030 & 0.039 & -0.032 & 0.034 & -0.031 & 0.049 & -0.006 & 0.053 & 0.104 & 0.003 & -0.041 \\
\hline $\begin{array}{l}\text { PC- } \\
\mathrm{O}(34: 1) \& \\
\mathrm{PC}- \\
\mathrm{P}(34: 0)\end{array}$ & 0.020 & -0.015 & 0.023 & -0.013 & 0.021 & 0.002 & 0.022 & -0.017 & 0.025 & -0.012 & 0.022 & -0.006 \\
\hline $\mathrm{SM}(36: 2)$ & 0.015 & -0.010 & 0.006 & -0.002 & 0.008 & -0.003 & 0.014 & 0.001 & 0.008 & 0.012 & 0.007 & 0.009 \\
\hline $\operatorname{SM}(34: 2)$ & 0.014 & 0.000 & 0.001 & 0.009 & 0.002 & 0.010 & 0.009 & 0.010 & 0.006 & 0.015 & 0.008 & 0.019 \\
\hline $\operatorname{SM}(32: 1)$ & 0.008 & -0.036 & 0.014 & 0.039 & 0.001 & 0.061 & 0.009 & 0.005 & 0.020 & 0.020 & 0.012 & 0.018 \\
\hline $\begin{array}{l}\text { PC- } \\
\mathrm{O}(36: 4) \& \\
\mathrm{PC}- \\
\mathrm{P}(36: 3)\end{array}$ & 0.003 & -0.002 & 0.001 & -0.001 & 0.004 & -0.004 & 0.005 & -0.004 & 0.007 & 0.009 & 0.002 & 0.006 \\
\hline $\operatorname{SM}(39: 1)$ & -0.009 & -0.008 & -0.011 & 0.011 & -0.013 & 0.016 & -0.007 & 0.001 & -0.006 & 0.005 & -0.009 & 0.017 \\
\hline
\end{tabular}

Table 1. Loading values for candidate biomarkers. Loading values generated by PCAs for the candidate biomarkers identified by sPLS-DA. Each exposed group (e.g. SGA-breastfed) tested against the appropriate control group (e.g. Control-breastfed). Loading values reflect the ranking of the variables by the (unsupervised) test. Note that the quantified rankings are only valid within principal components and thus cannot be compared between the groups. This test indicated that similar variables to those identified in Fig. 2 were important.
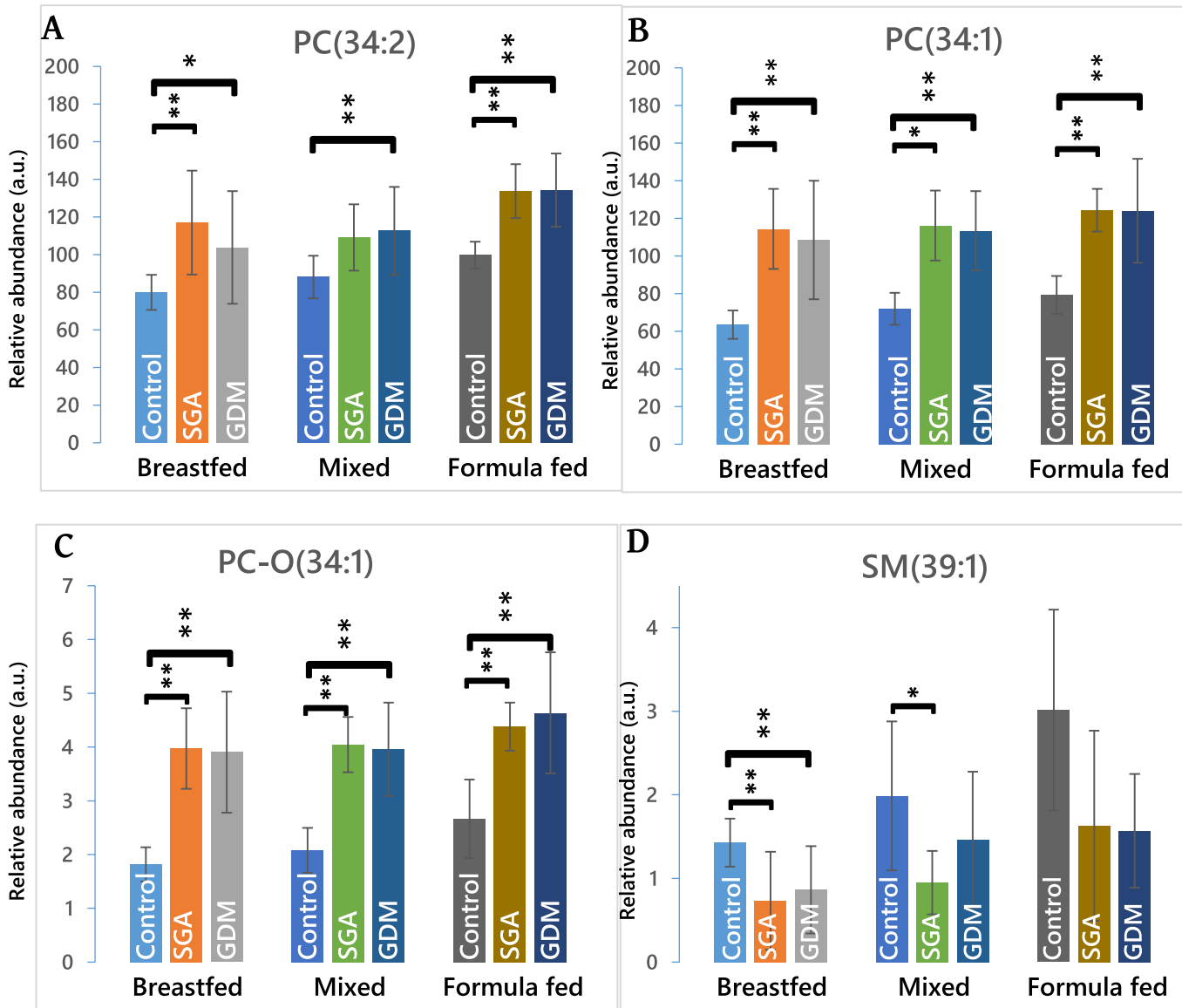

Figure 1. The abundance of candidate biomarkers in infants with normal conditions in utero as well as in SGA and MHG. Only these four lipids passed FDR based on a Bonferroni adjusted significance threshold. Panel (A) PC(34:2), isobaric with PE(37:2); (B) PC(34:1), isobaric with PE(37:1); (C) PC-O(34:1), isobaric with PC-P(34:0); (D) SM(39:1). No. variables=340. $p$-Values for Bonferroni corrected T-test: for dependent variables $=0.00271\left(\right.$ marked $\left.^{*}\right)$ and independent variables $=0.000147$ (marked $\left.* *\right)$ respectively. 

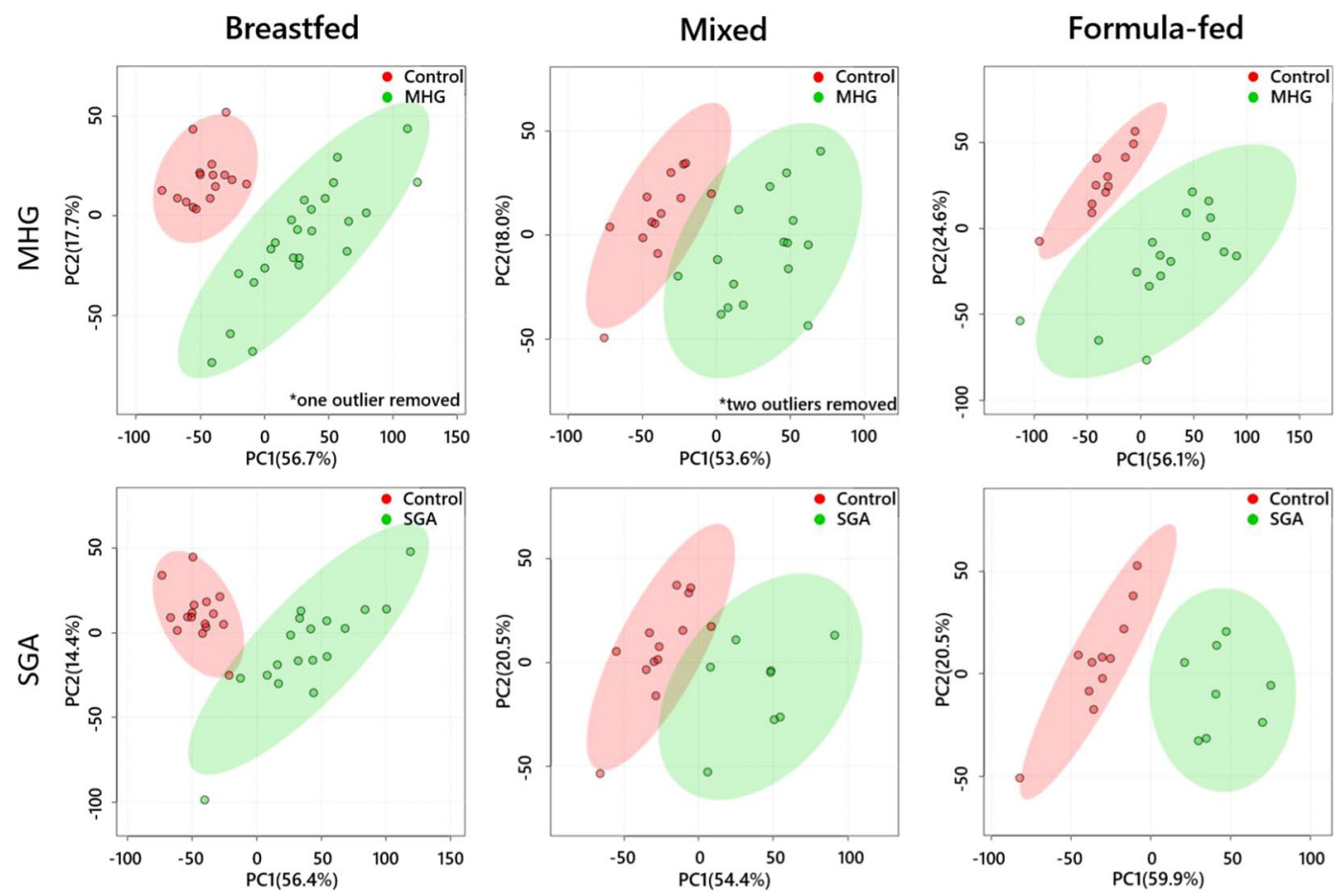

Figure 2. Principal Component Analyses of lipid profiles of infants born as SGA or from mothers with gestational diabetes (green) against controls (red).

Identification of candidate biomarkers (CBMs). In order to identify CBMs for differences in lipid metabolism from the variables identified by the sPLS-DA, the abundance of the molecular species must also be considered. The abundances of the variables across the various groups were calculated (mean of all values in each group) and T-tests applied in order to quantify the significance of the difference in the mean of abundance in exposed and control groups. The $p$-value cut offs used were 0.00271 for dependent variables and 0.000147 for independent variables. These tests identified PC(34:1), PC(34:2) and PC-O(34:1) particularly clearly (Fig. 1). The abundance of these phospholipids was higher in MHG and SGA groups compared to Controls, howsoever fed (Fig. 1 and Supplementary Table 1). SM(39:1) showed differences between in utero groups among breastfed infants, but these differences were not significant among mixed-fed or formula-fed infants. Among breast-fed infants only, differences between the three in utero groups were also seen in the abundance of PC(38:4) and SM(36:2), but these were not significant according to the Bonferroni adjusted threshold.

Notably, the abundances of PC(34:1), PC(34:2) and PC-O(34:1) were higher in both SGA and MHG compared to controls (Fig. 1). By contrast, the abundance of SM(39:1) is lower in SGA and MHG infants than in controls. In order to verify the results of the sPLS-DA and univariate tests, we performed an unsupervised analytical approach (principal component analysis, PCA) on the whole dataset. The PCAs (Fig. 2) showed that all subgroups of SGA and MHG infants were distinct from Control infants, across all feeding types. Furthermore, PC(34:1) and $\mathrm{PC}(34: 2)$ are the most important of these CBMs in distinguishing the groups, as they were more abundant than any other species measured (Fig. 1, full loadings shown in Supplementary Table 2).

\section{Discussion}

In this study, mass spectrometry was used to profile the phospholipids and triglycerides in the circulation of infants; statistical approaches were used to identify firstly variables and after more thorough testing candidate biomarkers (CBMs), that distinguish breastfed infants from those formula fed and conditions in utero simultaneously. These approaches identified $\mathrm{PC}(34: 1)$ and $\mathrm{PC}(34: 2)$ as the $\mathrm{CBMs}$ that represent the clearest difference in phospholipid metabolism, with $\mathrm{PC}-\mathrm{O}(34: 1)$ and SM(39:1) performing more moderately, but also being significant. However, all four of these lipids have the same head group (choline) and three of the four are very similar isoforms (34:1 or 34:2). This uniformity hints that these species may originate from a common process or under control of the same pathway. These species undoubtedly have structural roles in vivo, however, PCs are increasingly being recognised as having signalling functions as well, including these isoforms of $\mathrm{PC}^{31}$. In addition, SM(39:1) has recently been identified as a marker for dietary intake of fresh milk ${ }^{24}$. The PCs were more abundant in the circulation of infants from abnormal uterine conditions (SGA and MHG) than in Controls, whereas SM(39:1) was less abundant than controls across all feeding types.

Evidence collected over the last decade has shown that an isoform of $\mathrm{PC}(34: 1), \mathrm{PC}(16: 0 / 18: 1)$, has been identified as an endogenous ligand (agonist) for the nuclear receptor PPAR $\alpha$ (proxisome proliferator-activated receptor alpha), found in hepatocytes. PPAR $\alpha$ is a transcription factor regulating the expression of a number of genes involved in governing metabolism of triglycerides ${ }^{32}$ whose expression increases in foetal liver in pregnancies that progress normally ${ }^{33}$. Activation of PPAR $\alpha$ is observed under fasting conditions ${ }^{34}$ and is characterised by increases 


\begin{tabular}{|c|c|c|c|c|}
\hline & & Control & SGA & MHG \\
\hline \multirow[t]{4}{*}{$n$} & & 40 & 34 & 59 \\
\hline & Breastfed & 16 & 18 & 25 \\
\hline & Mixed feeding & 13 & 8 & 18 \\
\hline & Formula fed & 11 & 8 & 16 \\
\hline \multirow[t]{9}{*}{ Birth weight $(\mathrm{Kg})$} & & 39 & 34 & 59 \\
\hline & Mean & 3.37 & 2.41 & 3.3 \\
\hline & s.d. & 0.38 & 0.24 & 0.37 \\
\hline & Range (Lowest) & 2.65 & 1.86 & 2.72 \\
\hline & Range (Highest) & 4.02 & 2.85 & 4.88 \\
\hline & Significance & & & \\
\hline & against control group & - & 5.10E-09 & 0.63 \\
\hline & against SGA group & $5.10 \mathrm{E}-09$ & - & $5.10 \mathrm{E}-09$ \\
\hline & against MHG group & 0.63 & $5.10 \mathrm{E}-09$ & - \\
\hline \multirow[t]{9}{*}{ Gestational age (w) } & & 40 & 33 & 59 \\
\hline & Mean & 39.84 & 39.64 & 39.05 \\
\hline & s.d. & 1.33 & 1.61 & 0.94 \\
\hline & Range (Lowest) & 36.00 & 36.14 & 36.57 \\
\hline & Range (Highest) & 41.86 & 43.00 & 41.43 \\
\hline & Significance & & & \\
\hline & against control group & - & 0.76 & $7.04 \mathrm{E}-03$ \\
\hline & against SGA group & 0.76 & - & 0.08 \\
\hline & against MHG group & $7.04 \mathrm{E}-03$ & 0.08 & - \\
\hline \multirow{3}{*}{ No. male (\%) } & Breastfed & 31 & 50 & 32 \\
\hline & Mixed feeding & 62 & 38 & 78 \\
\hline & Formula-fed & 27 & 38 & 88 \\
\hline \multirow[t]{9}{*}{ Maternal age (years) } & & 40 & 33 & 59 \\
\hline & Mean & 32.89 & 33.19 & 34.53 \\
\hline & s.d. & 5.42 & 4.68 & 4.34 \\
\hline & Range (Lowest) & 19.53 & 23.15 & 22.69 \\
\hline & Range (Highest) & 41.33 & 43.56 & 44.14 \\
\hline & Significance & & & \\
\hline & against control group & - & 0.96 & 0.22 \\
\hline & against SGA group & 0.96 & - & 0.40 \\
\hline & against MHG group & 0.22 & 0.40 & - \\
\hline \multirow[t]{9}{*}{ Maternal BMI } & & 34 & 30 & 46 \\
\hline & Mean & 22.77 & 24.1 & 25.92 \\
\hline & s.d. & 4.29 & 4.88 & 6.05 \\
\hline & Range (Lowest) & 17.68 & 19.22 & 17.33 \\
\hline & Range (Highest) & 35.23 & 35.48 & 44.39 \\
\hline & Significance & & & \\
\hline & against control group & - & 0.57 & 0.02 \\
\hline & against SGA group & 0.57 & - & 0.30 \\
\hline & against MHG group & 0.02 & 0.30 & - \\
\hline
\end{tabular}

Table 2. Participant characteristics. The characteristics of the participants of the subset if the Cambridge Baby Growth Study II cohort used in this study. $n=133$. Significance was calculated using a Tukey HSD post-hoc analysis. Maternal BMI was taken at or before conception. Maternal age is as at delivery.

in the catabolism of fatty acids and the abundance of peroxisomes ${ }^{32}$. In adults, it also decreases hepatic steatosis ${ }^{35}$ but accelerates the development of the epidermal permeability barrier in the foetus ${ }^{36}$. PC(34:1) increases in abundance by up to $100 \%$ between the control and both MHG and SGA groups in this study (Fig. 1). This suggests that activation of PPAR $\alpha$ may be more common in the exposed groups. If true, one would therefore expect a faster rate of catabolism of fatty acids, and a weaker tendency of them being stored as triglycerides in SGA and MHG babies. An interaction such as this may form the basis for the metabolic programming effects observed by the conditions in utero and the feeding type afterwards.

We note that PC(34:1) appears as both a post natal nutritional CBM (i.e. for feeding type) and one that distinguishes conditions in utero. It is not clear from the present evidence whether this lipid is ubiquitous or has several functions. There is a plethora of evidence for a variety of phospholipids possessing even three or more functions simultaneously ${ }^{31,37,38}$. One possibility is that the structural differences between the control and exposed groups 
in this study have driven changes in the abundance of commonplace structural molecules such as PC, that also have other roles ${ }^{31}$.

The abundance of PC(18:0/18:1), an isoform of PC(36:1), is also different in breastfed infants in this study. The abundance of PC(36:1) is around 60\% higher in both SGA and MHG groups than normal birth weight Controls, among infants receiving breastfeeding (Table 3 ). However, the abundance is similar in across in utero groups fed formula (Table 3). PC(18:0/18:1) is an endogenous agonist for another PPAR, PPAR $\delta$, with activation reducing the concentration of triglycerides in plasma post prandium and increasing fatty acid use in the periph$e^{2} y^{39}$. However, there is also evidence that the two receptors are sufficiently specific such that PC(18:0/18:1) is not cross-reactivate with the receptor specific for $\mathrm{PC}(16: 0 / 18: 1)^{31}$.

These data therefore suggest that activation of $\mathrm{PPAR}_{\delta}$ may be different in babies receiving formula from those receiving breastmilk. If true, the triglycerides consumed by babies ingesting breastmilk will have a shorter half-life (be in the circulation for a shorter time) than those only fed formulae.

Furthermore, a naturally-occurring, endogenously-produced oxidised form of $\mathrm{PC}-\mathrm{O}(34: 1)$ is a potent agonist of PPAR $\gamma^{40}$. PPAR $\gamma$ is widely regarded as a key transcription factor in adipocyte differentiation ${ }^{41,42}$ and as $\mathrm{PC}-\mathrm{O}(34: 1)$ is much more abundant in infants from GDM pregnancies than controls, we might predict that more of the oxidised form (oxPC-O(34:1), which is commercially available) in circulo and that this leads to higher activation of PPAR $\gamma$ in SGA and MHG infants. This is significant because activation of PPAR $\gamma$ through an increase in the abundance of $\mathrm{PC}-\mathrm{O}(34: 1)$ provides a mechanism by which this species promotes adiposity. This may be important in long term metabolic health as there is now evidence that adiposity in adolescents is modulated by breastfeeding countering the deleterious effects of a PPAR $\gamma^{43}$.

More generally, how PPARs are expressed in the foetus can be influenced by maternal diet and obesity ${ }^{44,45}$. Specifically, an animal model has been shown that intra-uterine growth restriction decreases the expression of $\operatorname{PPAR} \gamma$ in the lung ${ }^{46}$ but increases it in adipocytes ${ }^{47}$.

The summative effect of both the putative increased abundance of agonists for PPAR $\alpha, \operatorname{PPAR} \delta$ and PPAR $\gamma$ and changes to their expression is unclear at present, however we expect a mixture of effects with respect to healthy systems as the catabolism of fatty acids and storage of TGs is favoured. This has implications for the relationship between conditions in utero and shortly thereafter, and later development. Further work is required to establish whether the CBMs identified in this study have a functional or metabolic programming influence on metabolism in infants who experienced different conditions in utero. This could be achieved by another study with increased statistical power but also follow-up samples and meta-data. Further work is also required to determine the origin and an understanding of how the abundance of such species is controlled, either by the infant's system or the maternal one.

The sPLS-DAs used to identify CBMs can also be used to assess how similar the individual members of the groups are to one another. For example, the formula-fed infants in both MHG and SGA groups appear to be far more homogeneous than either the mixed or breastfed groups (Supplementary Fig. 3). This raises questions about the variety of phospholipid and triglyceride profiles in breastmilk, and their relationship with the mothers' diet. A larger sample size in a different group of individuals is required to investigate this. However, these results suggest that the metabolic activity in formula-fed SGA and MHG infants is more homogeneous than other groups, hinting that a narrower range of factors shapes the lipid profile found in their circulation.

Lastly, recent work on the lipid and triglyceride composition of infant formula suggests that this type of dietary intake varies considerably by manufacturer, manufacturing site and target demographic ${ }^{48}$. This may explain some of the heterogeneity in the groups fed formula. Several of the most abundant phospholipid species were similar across formulae, though the triglyceride profiles of formula and fresh milk were distinct. This suggests that further study of the relationship between lipid metabolism following the feeding of infant formula, and in a high powered manner, is required.

The present study represents a pilot, mechanistic-type investigation of the relationship between conditions in utero and later feeding. To our knowledge it is the first of its kind. It is limited in the manner that is typical of pilot studies (e.g. modest statistical power). Recent work on infant formula has shown that it is a varied and complicated mixture, suggesting that the traditional classification of 'formula-fed' used in the present study and many others is a general one. However, the present study is capable of suggesting which of the comparisons are more important and thus which are of principal interest for further studies, both clinical and mechanistic.

\section{Conclusion}

In this study, the hypothesis that the lipid and triglyceride metabolism in infants is shaped by antenatal conditions (including glucose supply) and postnatal nutrition was tested in a pilot cohort by profiling the phospholipid, triglyceride and sterol fractions in the circulation of infants three months post partum. Three separate statistical methods indicate that the abundance of a small number of phospholipids with a narrow range of structures (PC34:1), PC(34:2) and PC-O(34:1)) best distinguishes the groups of individuals. We note that phospholipids with this molecular structure have an important role in triglyceride metabolism in humans, through interaction with PPARs. Furthermore, the evidence from the separate analyses in this study indicates that the feeding type applied to infants is as important for the phospholipid profile observed in infants at three months post partum as the effect of the in uterine conditions associated with SGA and MHG. This study therefore provides evidence that both feeding and conditions in utero shape short-term phospholipid metabolism in early life. Furthermore, the similar nature of the pattern of abundance of CBMs between exposed groups (SGA and MHG) indicate that the shifts in lipid metabolism may be similar between these groups.

The precise role or effects in infants of the candidate biomarkers identified in this study is not yet fully understood. Further work may explore how changes in phospholipid metabolism can be used to correct the metabolic consequences of unfavourable conditions in utero. 


\begin{tabular}{|l|l|l|l|l|l|}
\hline & & & & \multicolumn{2}{l}{ Loadings values } \\
\cline { 5 - 6 } & Mean & StDev & T-test & PC1 & PC2 \\
\hline Control (BF) & 14.072 & 2.250 & & & \\
\hline SGA (BF) & 25.106 & 4.530 & $1.573 \times 10^{-9}$ & 0.1026 & -0.0862 \\
\hline MHG (BF) & 25.070 & 7.010 & $6.370 \times 10^{-8}$ & 0.1270 & -0.0833 \\
\hline Control (M) & 15.099 & 2.054 & & & \\
\hline SGA (M) & 26.355 & 3.995 & $3.440 \times 10^{-5}$ & 0.1356 & -0.0806 \\
\hline MHG (M) & 24.438 & 4.271 & $1.545 \times 10^{-8}$ & 0.1189 & -0.0754 \\
\hline Control (FF) & 15.415 & 2.706 & & & \\
\hline SGA (FF) & 14.230 & 2.172 & $3.409 \times 10^{-7}$ & 0.1284 & 0.0072 \\
\hline MHG (FF) & 14.072 & 2.250 & $1.701 \times 10^{-8}$ & 0.0947 & -0.0588 \\
\hline
\end{tabular}

Table 3. Abundance of $\mathrm{PC}(36: 1)$. Mean and standard deviation of abundance of $\mathrm{PC}(36: 1)$, $\mathrm{p}$-value associated with the difference between exposed and control samples and the loadings in the PCAs (Fig. 2). PC(36:1) is not a CBM due to the similar abundance across formula fed groups. BF, breastfed; $M$, mixed feeding; FF, formulafed.

\section{Experimental Methods}

Study design. This pilot study was designed to determine what, if any, differences in phospholipid, sterol and/or triglyceride metabolism there are between infants as a factor of both conditions in utero and feeding in the first three months of life. This necessitated a suitable array of participants, consistent sample collection, detailed measurement of molecular abundance and statistical analyses.

Cohort. We used a subset of infant participants from the Cambridge Baby Growth Study II, characterised in Table 2. Only samples from singleton births were used. This is a prospective observational birth cohort, focussing on the antenatal and early postnatal determinants of infancy growth (22) Mothers were recruited during early pregnancy from a single antenatal centre in Cambridge.

There were three groups of infant participants according to conditions in utero: (a) infants being born small for gestational age (SGA group), (b) a normal birth weight (Control group) and (c) a normal birth weight group who had exposed to maternal hyperglycaemia (MHG group; mothers of infants in this group were diagnosed according to IADPSG thresholds for GDM $)^{49}$. There were three groups within each of these in utero groups, based on feeding mode. Feeding groups were of breastfed, formula-fed or infants receiving a mixed intake (Table 2). We were not able to determine the relative proportions of the two due to well-known experimental difficulties in establishing the volume of milk consumed during breast feeding.

Ethics. All procedures were performed in accordance with the ethical standards of the institutional and/or national research committee and with the 1964 Helsinki declaration and its later amendments or comparable ethical standards. All mothers gave informed written consent and the study was approved by the Cambridge local research ethics committee (LREC 11/EE/0068). Class membership of samples were blinded and so technicians and analysts were not aware of which participants fell into which groups until after the lipid profiles were available, and then only by participant number.

Reagents and standards. Solvents of at least HPLC grade were purchased from Sigma-Aldrich Ltd. (Gillingham, Dorset, UK) and were not purified further. Lipid standards were purchased from Avanti Polar lipids (Alabaster, AL; through Instruchemie, Delfzijl, NL) and used without purification. Consumables were purchased from Sarstedt AG \& Co (Leicester, UK).

Sample collection. Samples from the three in utero groups were drawn from infants $(n=133)$ who participated in the Cambridge Baby Growth Study II and collected between 2011 and 2013. Their infants were seen at birth by trained research nurses and followed-up at 3 months. Blood was collected by a heel prick, spotted immediately on untreated filter paper cards (Ahlstrom 226; ID Biological Systems, Greenville, South Carolina) and dried at room temperature overnight. Dried blood spots were stored under a small, sealed air atmosphere at $-80^{\circ} \mathrm{C}$. Samples were collected during morning appointments (0900-1130). Infancy feeding (exclusive breast-, mixed or exclusive formula-feeding) was assessed by questionnaire at age 3 months.

Lipid extraction. The phospholipid, triglyceride and sterol fractions were isolated together using a method reported recently ${ }^{50}$. Briefly, dried blood spot samples, as $3.2 \mathrm{~mm}$ diameter discs, were placed along with blank and Quality Control samples (QCs) in the wells of a glass coated $2.4 \mathrm{~mL} /$ well ninety-six-well plate (96w plate; Plate $+{ }^{\mathrm{TM}}$, Esslab, Hadleigh, UK). Water $(100 \mu \mathrm{L}$, MilliQ) was added to each of the wells, followed by methanol $(250 \mu \mathrm{L}$, HPLC grade, spiked with $0.6 \mu \mathrm{M}$ 1,2-di-O-octadecyl-sn-glycero-3-p hosphocholine, $1.2 \mu \mathrm{M}$ 1,2-di-O-phytanyl-sn-glycero-3-phosphoethanolamine, $0.6 \mu \mathrm{M} \mathrm{C}_{8}$-ceramide, $0.6 \mu \mathrm{M}$ $N$-heptadecanoyl-D-erythro-sphingosylphosporylcholine, $6 \cdot 2 \mu \mathrm{M}$ undecanoic acid, $0.6 \mu \mathrm{M}$ trilaurin), followed by tert-butyl methyl ether (TMBE, $500 \mu \mathrm{L}$ ). The plates were then sealed (aluminium microplate sealing tape), agitated $(10 \mathrm{~min}, 600 \mathrm{rpm})$ and centrifuged for $10 \mathrm{~min}$ at $3.2 \mathrm{k} \times \mathrm{g}$. A 96 -head micro-dispenser was used to transfer $200 \mu \mathrm{L}$ of the organic solution to a glass-coated $240 \mu \mathrm{L} 96 \mathrm{w}$ plate (Plate $+{ }^{\mathrm{TM}}$, Esslab, Hadleigh, UK). The plate was transferred to a Genevac EZ-2 evaporator (Genevac Ltd., Ipswich, UK) and dried. The samples were reconstituted 
(TBME, $25 \mu \mathrm{L}$ and MS-mix [7.5 mM ammonium acetate in IPA: $\mathrm{CH}_{3} \mathrm{OH}(2: 1)$ ], $90 \mu \mathrm{L}$ ) of using a Hydra Matrix, after which the plate was sealed and stored at $-20^{\circ} \mathrm{C}$.

Mass spectrometry. All samples were infused into an Exactive Orbitrap (Thermo, Hemel Hampstead, UK), using a Triversa Nanomate (Advion, Ithaca US) and a mass resolution of 100,000. Samples were ionised at $1 \cdot 2 \mathrm{kV}$. The Exactive started acquiring data $20 \mathrm{~s}$ after sample aspiration began. After $72 \mathrm{~s}$ of acquisition in positive mode the Nanomate and the Exactive switched to negative mode, decreasing the voltage to $-1.5 \mathrm{kV}$. The spray was maintained for another $66 \mathrm{~s}$, after which the analysis was stopped and the tip discarded, before the analysis of the next sample began. Throughout the analysis the sample plate was kept at $15^{\circ} \mathrm{C}$. Samples were run in row order.

The phospholipid and triglyceride signals obtained were relative abundance ('semi-quantitative'), with the signal intensity of each lipid expressed relative to the total lipid signal intensity, for each individual, per cent (\%). Raw high-resolution mass-spectrometry data were processed using XCMS (www.bioconductor.org) and Peakpicker v 2.0 (an in-house R script).

Statistical methods. In order to identify which individual molecular species best represented the differences in the lipid metabolism between the various groups, we employed several statistical methods. All multivariate analyses (MVA) except Random Forest were carried out using Metaboanalyst $4.0^{51}$. Random Forest classifications were carried out using R. Univariate tests were carried out using Microsoft Excel 2013. Significance thresholds were corrected for multiple testing and $p$-value cut offs used were 0.00271 for dependent variables and 0.000147 for independent variables.

The first stage of our analysis was to augment a previous analysis with the lipid profile collected in negative ionisation mode. The previous analysis used species detected in positive mode only ${ }^{25}$. This employed the same unsupervised test used in previous work, viz. a Principal Component Analysis (PCA). This indicated a fuller range of detectable molecular species that were associated with feeding type. The second stage was designed to test whether conditions in utero (SGA, MHG, Control) had an effect on lipid metabolism that was generally independent of that of feeding. A Random Forest classification was used for this test, using three groups and 10k trees. The third stage was candidate biomarker discovery. This comprised two parts. First, a sparse Partial Least Squares Discriminant Analysis was used to identify individual variables that distinguished the groups from one another, followed by (univariate) T-tests to identify which candidate biomarkers (CBMs) were significantly different in abundance. Second, an unsupervised MVA statistical test was used to verify the ranked importance of the CBMs identified in the first stage (supervised MVA and univariate tests). The type of MVA was unsupervised (PCA) because this offers an orthogonal approach to identifying variables but also to minimise bias.

\section{Data Availability}

The datasets generated during and analysed during the current study are available from the corresponding author on reasonable request.

\section{References}

1. Blackmore, H. L. et al. Maternal Diet-induced Obesity Programs Cardiovascular Dysfunction in Adult Male Mouse Offspring Independent of Current Body Weight. Endocrinology 155, 3970-3980, https://doi.org/10.1210/en.2014-1383 (2014).

2. Loche, E. et al. Maternal diet-induced obesity programmes cardiac dysfunction in male mice independently of post-weaning diet. Cardiovascular Research 114, 1372-1384, https://doi.org/10.1093/cvr/cvy082 (2018).

3. Alfaradhi, M. Z. et al. Maternal Obesity in Pregnancy Developmentally Programs Adipose Tissue Inflammation in Young, Lean Male Mice Offspring. Endocrinology 157, 4246-4256, https://doi.org/10.1210/en.2016-1314 (2016).

4. Duque-Guimarães, D. E. \& Ozanne, S. E. Nutritional programming of insulin resistance: causes and consequences. Trends in Endocrinology \& Metabolism 24, 525-535, https://doi.org/10.1016/j.tem.2013.05.006 (2013).

5. Samuelsson, A.-M. et al. Diet-Induced Obesity in Female Mice Leads to Offspring Hyperphagia, Adiposity, Hypertension, and Insulin Resistance. Hypertension 51, 383-392, https://doi.org/10.1161/HYPERTENSIONAHA.107.101477 (2008).

6. Shelley, P. et al. Altered skeletal muscle insulin signaling and mitochondrial complex II-III linked activity in adult offspring of obese mice. American Journal of Physiology-Regulatory, Integrative and Comparative Physiology 297, R675-R681, https://doi.org/10.1152/ aipregu.00146.2009 (2009).

7. Oben, J. A. et al. Maternal obesity during pregnancy and lactation programs the development of offspring non-alcoholic fatty liver disease in mice. Journal of Hepatology 52, 913-920, https://doi.org/10.1016/j.jhep.2009.12.042 (2010).

8. Musial, B. et al. A Western-style obesogenic diet alters maternal metabolic physiology with consequences for fetal nutrient acquisition in mice. The Journal of Physiology 595, 4875-4892, https://doi.org/10.1113/JP273684 (2017).

9. Fernandez-Twinn, D. S. et al. Exercise rescues obese mothers' insulin sensitivity, placental hypoxia and male offspring insulin sensitivity. Scientific Reports 7, 44650, https://doi.org/10.1038/srep44650 (2017).

10. Brooke, O. G., Anderson, H. R., Bland, J. M., Peacock, J. L. \& Stewart, C. M. Effects on birth weight of smoking, alcohol, caffeine, socioeconomic factors, and psychosocial stress. British Medical Journal 298, 795-801, https://doi.org/10.1136/bmj.298.6676.795 (1989).

11. Kramer, M. S. Determinants of low birth weight: methodological assessment and meta-analysis. Bulletin of the World Health Organization 65, 663-737 (1987).

12. Martin, T. R. \& Bracken, M. B. The Association Between Low Birth Weight and Caffeine Consumption During Pregnancy. American Journal of Epidemiology 126, 813-821, https://doi.org/10.1093/oxfordjournals.aje.a114718 (1987).

13. Spellacy, W. N., Miller, S., Winegar, A. \& Peterson, P. Q. Macrosomia-maternal characteristics and infant complications. Obstet Gynecol 66, 158-161 (1985)

14. Clausen, T. et al. Maternal anthropometric and metabolic factors in the first half of pregnancy and risk of neonatal macrosomia in term pregnancies. A prospective study. European Journal of Endocrinology 153, 887-894, https://doi.org/10.1530/eje.1.02034 (2005).

15. Khashan, A. S. \& Kenny, L. C. The effects of maternal body mass index on pregnancy outcome. European Journal of Epidemiology 24, 697, https://doi.org/10.1007/s10654-009-9375-2 (2009).

16. Gillman, M. W., Rifas-Shiman, S., Berkey, C. S., Field, A. E. \& Colditz, G. A. Maternal Gestational Diabetes, Birth Weight, and Adolescent Obesity. Pediatrics 111, e221-e226, https://doi.org/10.1542/peds.111.3.e221 (2003). 
17. Kc, K., Shakya, S. \& Zhang, H. Gestational Diabetes Mellitus and Macrosomia: A Literature Review. Annals of Nutrition and Metabolism 66(suppl 2), 14-20, https://doi.org/10.1159/000371628 (2015).

18. Vally, F., Presneill, J. \& Cade, T. Macrosomia Rates in Women with Diet-Controlled Gestational Diabetes: A Retrospective Study. Journal of Pregnancy 2017, 5, https://doi.org/10.1155/2017/4935397 (2017).

19. Van Assche, F. A., Holemans, K. \& Aerts, L. Long-term consequences for offspring of diabetes during pregnancy. British Medical Bulletin 60, 173-182, https://doi.org/10.1093/bmb/60.1.173 (2001).

20. Yao, R., Park, B. Y. \& Caughey, A. B. The effects of maternal obesity on perinatal outcomes among those born small for gestational age*. The Journal of Maternal-Fetal \& Neonatal Medicine 30, 1417-1422, https://doi.org/10.1080/14767058.2016.1216098 (2017).

21. Robillard, P.-Y., Dekker, G., Boukerrou, M., Le Moullec, N. \& Hulsey, T. C. Relationship between pre-pregnancy maternal BMI and optimal weight gain in singleton pregnancies. Heliyon 4, e00615, https://doi.org/10.1016/j.heliyon.2018.e00615 (2018).

22. Wei, J.-N. et al. Low Birth Weight and High Birth Weight Infants Are Both at an Increased Risk to Have Type 2 Diabetes Among Schoolchildren in Taiwan. Diabetes Care 26, 343-348, https://doi.org/10.2337/diacare.26.2.343 (2003).

23. Tsadok, M. A. et al. Obesity and blood pressure in 17-year-old offspring of mothers with gestational diabetes: insights from the Jerusalem Perinatal Study. Experimental diabetes research 2011, 906154-906154, https://doi.org/10.1155/2011/906154 (2011).

24. Acharjee, A. et al. The translation of lipid profiles to nutritional biomarkers in the study of infant metabolism. Metabolomics 13, 25, https://doi.org/10.1007/s11306-017-1166-2 (2017).

25. Prentice, P. et al. Lipidomic Analyses, Breast- and Formula-Feeding, and Growth in Infants. The Journal of Pediatrics 166, 276-281, https://doi.org/10.1016/j.jpeds.2014.10.021 (2015).

26. Furse, S., Eriksen, K. G., Moore, S. E. \& Koulman, A. Identification of candidate molecular biomarkers for growth faltering in infants at $12 \mathrm{w}$. In prep (2019).

27. Farrar, D. et al. Chapter 4-Prevalence of gestational diabetes in the UK and Republic of Ireland: a systematic review from the identification and treatment of women with hyperglycaemia in pregnancy: an analysis of individual participant data, systematic reviews, meta-analyses and an economic evaluation. Health technology assessment 20(86), 1-348 (2016).

28. Bodnar, L. M. et al. Maternal Serum 25-Hydroxyvitamin D Concentrations Are Associated with Small-for-Gestational Age Births in White Women. The Journal of Nutrition 140, 999-1006, https://doi.org/10.3945/jn.109.119636 (2010).

29. Saenger, P., Czernichow, P., Hughes, I. \& Reiter, E. O. Small for Gestational Age: Short Stature and Beyond. Endocrine Reviews 28, 219-251, https://doi.org/10.1210/er.2006-0039 (2007).

30. Albertsson-Wikland, K., Wennergren, G., Wennergren, M., Vilbergsson, G. \& Rosberg, S. Longitudinal follow-up of growth in children born small for gestational age. Acta Paediatrica 82, 438-443, https://doi.org/10.1111/j.1651-2227.1993.tb12718.x (1993),

31. Furse, S. \& de Kroon, A. I. P. M. Phosphatidylcholine's functions beyond that of a membrane brick. Molecular Membrane Biology 32, 117-119, https://doi.org/10.3109/09687688.2015.1066894 (2015).

32. Kersten, S. Integrated physiology and systems biology of PPAR $\alpha$. Molecular Metabolism 3, 354-371, https://doi.org/10.1016/j. molmet.2014.02.002 (2014)

33. Abbott, B. D., Wood, C. R., Watkins, A. M., Das, K. P. \& Lau, C. S. Peroxisome Proliferator-Activated Receptors Alpha, Beta, and Gamma mRNA and Protein Expression in Human Fetal Tissues. PPAR Research 2010, 690907, https://doi.org/10.1155/2010/690907 (2010).

34. Kersten, S. et al. Peroxisome proliferator-activated receptor $\alpha$ mediates the adaptive response to fasting. The Journal of Clinical Investigation 103, 1489-1498, https://doi.org/10.1172/JCI6223 (1999).

35. Chakravarthy, M. V. et al. Identification of a Physiologically Relevant Endogenous Ligand for PPAR $\alpha$ in Liver. Cell 138, 476-488, https://doi.org/10.1016/j.cell.2009.05.036 (2009).

36. Hanley, A. J. G., Johnson, K. C., Villeneuve, P. J. \& Mao, Y. Canadian Cancer Registries Epidemiology Research, G. Physical activity, anthropometric factors and risk of pancreatic cancer: Results from the Canadian enhanced cancer surveillance system. International Journal of Cancer 94, 140-147, https://doi.org/10.1002/ijc.1446 (2001).

37. Furse, S. Is phosphatidylglycerol essential for terrestrial life? Journal of Chemical Biology 10, 1-9, https://doi.org/10.1007/s12154016-0159-3 (2017).

38. Furse, S. \& Shearman, G. C. Do lipids shape the eukaryotic cell cycle? Biochimica et Biophysica Acta 1863, 9-19, https://doi. org/10.1016/j.bbalip.2017.09.010 (2018)

39. Liu, S. et al. A diurnal serum lipid integrates hepatic lipogenesis and peripheral fatty acid use. Nature 502, 550-554, https://doi. org/10.1038/nature12710 (2013).

40. Davies, S. S. et al. Oxidized Alkyl Phospholipids Are Specific, High Affinity Peroxisome Proliferator-activated Receptor $\gamma$ Ligands and Agonists. Journal of Biological Chemistry 276, 16015-16023, https://doi.org/10.1074/jbc.M100878200 (2001).

41. Berger, J. P. \& Moller, D. E. The Mechanisms of Action of PPARs. Annual Review of Medicine 53, 409-435, https://doi.org/10.1146/ annurev.med.53.082901.104018 (2002).

42. Grygiel-Górniak, B. Peroxisome proliferator-activated receptors and their ligands: nutritional and clinical implications - a review. Nutrition Journal 13, 17, https://doi.org/10.1186/1475-2891-13-17 (2014).

43. Verier, C. et al. Breast-Feeding Modulates the Influence of the Peroxisome Proliferator-Activated Receptor- $\gamma$ (PPARG2) Pro12Ala Polymorphism on Adiposity in Adolescents. The Healthy Lifestyle in Europe by Nutrition in Adolescence (HELENA) cross-sectional study 33, 190-196, https://doi.org/10.2337/dc09-1459 (2010).

44. Rees, W. D., McNeil, C. J. \& Maloney, C. A. The Roles of PPARs in the Fetal Origins of Metabolic Health and Disease. PPAR Research 2008, 8, https://doi.org/10.1155/2008/459030 (2008).

45. Desai, M. \& Ross, M. G. Fetal Programming of Adipose Tissue: Effects of Intrauterine Growth Restriction and Maternal Obesity/ High-Fat Diet. Semin Reprod Med 29, 237-245, https://doi.org/10.1055/s-0031-1275517 (2011).

46. Joss-Moore, L. A. et al. IUGR decreases PPAR $\gamma$ and SETD8 Expression in neonatal rat lung and these effects are ameliorated by maternal DHA supplementation. Early Human Development 86, 785-791, https://doi.org/10.1016/j.earlhumdev.2010.08.026 (2010).

47. Desai, M., Guang, H., Ferelli, M., Kallichanda, N. \& Lane, R. H. Programmed upregulation of adipogenic transcription factors in intrauterine growth-restricted offspring. Reprod Sci 15, 785-796, https://doi.org/10.1177/1933719108318597 (2008).

48. Furse, S. \& Koulman, A. The Lipid and Glyceride Profiles of Infant Formula Differ by Manufacturer, Region and Date Sold. Nutrients $11,1122(2019)$

49. IADPSG \& (Metzger, B. E). International Association of Diabetes and Pregnancy Study Groups Recommendations on the Diagnosis and Classification of Hyperglycemia in Pregnancy. Diabetes Care 33, 676-682, https://doi.org/10.2337/dc09-1848 (2010).

50. Koulman, A. et al. The development and validation of a fast and robust dried blood spot based lipid profiling method to study infant metabolism. Metabolomics 10, 1018-1025, https://doi.org/10.1007/s11306-014-0628-z (2014).

51. Chong, J. et al. MetaboAnalyst 4.0: towards more transparent and integrative metabolomics analysis. Nucleic Acids Research 46, W486-W494, https://doi.org/10.1093/nar/gky310 (2018).

\section{Acknowledgements}

The authors would like to thank the BBSRC for funding (BB/M027252/1 for SF and AK, and BB/P028195/1 for AK and SGS). The Cambridge Baby Growth Study has also been supported by the European Union Framework 5 (QLK4-1999-01422), the World Cancer Research Foundation International (2004/03), the Medical Research Council (7500001180), the NIHR Cambridge Comprehensive Biomedical Research Centre (NIHR 146281 for 
AK), the Newlife Foundation for disabled children (07/20), the Mothercare Charitable Foundation (RG54608), and Mead Johnson Nutrition. PP was supported by a MRC Clinical Training Fellowship (G1001995). KO is supported by the Medical Research Council (Unit Programme number: MC_UU_12015/2). The authors would like to thank Prof Susan Ozanne for helpful conversations and CBGS research nurses Suzanne Smith, Ann-Marie Wardell, and Karen Forbes for sample collection.

\section{Author Contributions}

S.F. designed its hypothesis for the present study, extracted and analysed the negative ion mode MS data carried out all statistical tests but the random forest and wrote the paper. S.G.S. carried out the random forest classifications and advised on other statistical tests. L.O. and P.P. collected samples and all meta-data. I.A.H. and D.B.D. conceived the CBGSs, and with K.K.O. wrote the grant proposals, and with C.L.A. managed the studies and made meta-data available. A.K. extracted the analytes and ran all samples, extracted positive ion mode data, provided critical review of the manuscript, and conceived and wrote the grant proposals that pay for S.F. and S.G.S.'s positions. All authors have read and approved the manuscript.

\section{Additional Information}

Supplementary information accompanies this paper at https://doi.org/10.1038/s41598-019-50693-0.

Competing Interests: The authors declare no competing interests.

Publisher's note Springer Nature remains neutral with regard to jurisdictional claims in published maps and institutional affiliations.

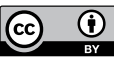

Open Access This article is licensed under a Creative Commons Attribution 4.0 International License, which permits use, sharing, adaptation, distribution and reproduction in any medium or format, as long as you give appropriate credit to the original author(s) and the source, provide a link to the Creative Commons license, and indicate if changes were made. The images or other third party material in this article are included in the article's Creative Commons license, unless indicated otherwise in a credit line to the material. If material is not included in the article's Creative Commons license and your intended use is not permitted by statutory regulation or exceeds the permitted use, you will need to obtain permission directly from the copyright holder. To view a copy of this license, visit http://creativecommons.org/licenses/by/4.0/.

(c) The Author(s) 2019 